\title{
19 \\ CALL PROCESSING MODEL FOR MULTIMEDIA SERVICES
}

\author{
Olli Martikainen \\ Telecom Finland Ltd. \\ P.O. Box 106, FIN-00511, Helsinki, Finland \\ Phone: +358 2040 3503, Fax: +3582040 3251 \\ E-mail: olli.martikainen@tele.telebox.fi
}

Valeri Naoumov, Konstantine Samouylov

Russian University of Peoples' Friendship

P.O. Box 9, 117419 Moscow, Russia

Phone: +7 095955 \{0863, 0956\}, Fax: +7 0959522823

E-mail: \{vnaoumov,ksam\}@udn.msk.su

\begin{abstract}
To support a wide range of multimedia services a B-ISDN network capabilities should allow the distribution of service functions between the customer terminals and the network nodes. Intelligent networks (IN) can be considered as the bridging technology between the customer based service management and the service execution in the networks. Considering the mobile and broadband technologies it is a natural question, how the concept of intelligence will develop. To satisfy broadband mobile and multimedia service needs IN should support much more distributed operations. It will undoubtedly lead to the increase of the volume of signaling messages. To carry a large number of messages IN must use enhanced call processing model instead of current Basic Call Model. In the paper a distributed call processing model for multimedia services for broadband intelligent networks is proposed and argued for.
\end{abstract}

\section{INTRODUCTION}

With the research on multimedia and broadband mobile services there arises a need to control complex combinations of service components and resources. Examples of these services are 
digital interactive TV, video on demand services for banking, shopping and leisure, electronic press and publishing. The technological requirements for these services are cost effective broadband transmission and access technologies, flexible computer based management and control of networks, switching and service applications and the support of mobility. The broadband transmission and switching technology is also maturing and will provide a cost effective platform for service provision. When the broadband customer access will be available, then interactive business and consumer services based on video and multimedia will become possible. Common to all these developments will be the computer controlled structure of modern services, where protocols, application technology and resource management are key factors.

In modern telephony the most promising computer control and management concept is the Intelligent Network (IN). It facilitates the development and management of new services by service providers. Intelligent networks can be considered as the bridging technology between the customer based service management and the service execution in the networks. It seems to be clear that the service applications will be distributed over terminals and different service control and provider nodes. An example of distributed intelligence can be found in the specifications of Universal Mobile Telecommunications Services [UMTS] and the Advanced Intelligent Mobile Communications Network [AIMN]. Different possibilities to introduce and distribute intelligence in future networks have been considered in [FUTU] and [LOCA].

A number of modifications and improvements to IN and call processing are necessary before future mobile telecommunication systems can be implemented. In this paper we propose a distributed call processing model for multimedia services in broadband intelligent networks.

\section{MULTIMEDIA SERVICES}

Multimedia service is a service in which the interchanged information consists of more than one type (e.g. video, data, voice, graphics). Monomedia communications related to single information types are described in a parts of a multimedia service called service components. Multimedia services may offer several virtual connections, one virtual connection for each service component, and each of the virtual connections may have particular QOS attribute values [AUNI], [I.374].

The service provision mechanisms would become in the future different from those of today.

The subscriber subscribes to and pays for services offered by one or more service providers on behalf of the real user of the services (customer). It is possible that the customer and the subscriber are the same person. On the other hand various subscribers could be linked to the same customer.

The terminal can be available for the use by many users and provides a level of service that is customized to the user. This implies that each customer must be identified when accessing to the network. The content control allows the customer to influence the content of the service. The customer can manipulate on-line the quality of the receiving service component by the modification of QOS parameters during the connection life time. Different multimedia serv- 
ices may require a service negotiation by the involved terminal to check if they can serve the same type of multimedia service or not.

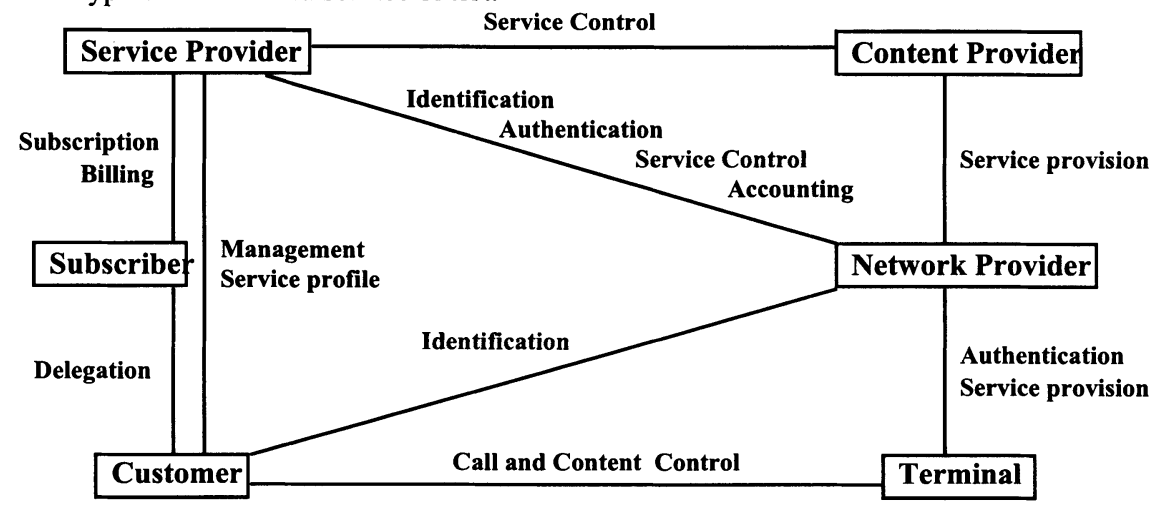

Figure 1 Multimedia service provision model.

The network provider provides standardized network services including security access to the network, individual connection links for a session and management of the service provider directory. The main function of a service provider is to design the interface for the customer. This includes the customer registration and identification, easy to use graphical interface, management of customer service profiles and billing/charging for the service. It performs also the session management function and end-to end service control. Service provider is responsible for agreements with network provider on network usage.

The primary function of a content provider is the storage and playback of multimedia content. For interactive services, it controls the playback content based on direct real-time interactions with the customer. This control may require access to and interaction with the network provided by the service provider.

\section{MOBILITY AND IN}

It is expected that the majority of new annual telephone subscriptions will be mobile-based after 1997. International Mobile Telecommunications 2000, IMT2000, formerly known as Future Public Land Mobile Telecommunication System, FPLMTS, should provide mobile telecommunications that support universal roaming and offer broadband multimedia services. To achieve in IMT2000 the Universal Mobile Telecommunication System, UMTS, is specified by ETSI [UMTS], and the Advanced Intelligent Mobile Communications Network, AIMN, is developed by NTT [AIMN]. In mobile services the service mobility is supported by the user location updating, which is a non-call associated function, and by handovers during the call. In handover the bearer connections are transfered from a base station to another depending on the best quality available in the adjacent radio transmission cells. 
The primary objective of IMT2000 is the service portability. It is obtained by terminal mobility and personal mobility. In addition to the basic IN Call Control Access Function (CCAF) for user call access, Call Control Function (CCF) for call processing control and Service Switching Function (SSF) for interaction between CCF and Service Control Function (SCF) [Q.1204], new functional entities have become necessary.

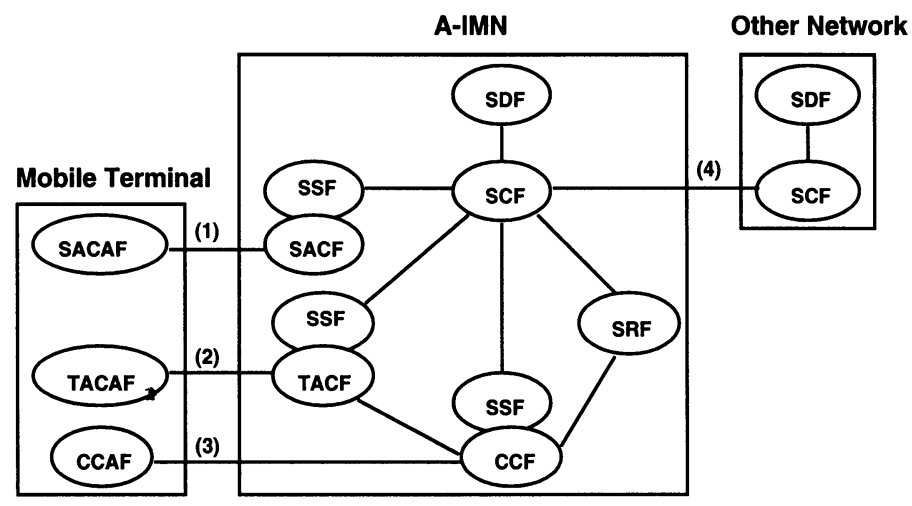

(1) - Non-Call/Non-Bearer Access Control Relationship

(2) - Terminal Access Control Relationship

(3) - Call Control Access Relationship

(4) - Inter-Network Control Relationship

Figure 2 IN Distributed Functional Plane Architecture introduced by A-IMN [AIMN].

Terminal mobility means the possibility to access services at anytime and anywhere. It implies terminal access control including terminal paging and terminal authentication for the authorization of the right to establish the bearer connection. For this control two new IN functional entities have been introduced: Terminal Access Control Function (TACF) and Terminal Access Control Agent Function (TACAF).

Personal mobility allows the identified customer to access services from any terminal. Several identification mechanisms can be used, e.g. manual PIN entry, biometric identification and smart card based identification [DATA].

Service profiles for service portability must be handled according to customer requests. Such non-call and non-bearer control includes terminal contation updating, customer registration, and service profile interrogation/modification. For this control Service Access Control Function (SACF) and Service Access Control Agent Function (SACAF) functional entities have been introduced. 


\section{MULTIMEDIA AND IN}

Current IN call processing functions representation called the Basic Call Model must be enhanced to support much more distributed operations and events taking place outside a call. A multimedia call model should support the ability to have several connections of the same or different types between any parties involved in a call. It has been widely agreed that future signaling protocols should support Call Control (CC) and Bearer Control (BC) separation [ISCP].

The Call Control is responsible for the establishment of an end-to-end communication configuration for users without allocating expensive network resources. No transit switches are involved in the Call Control. The Bearer (Connection) Control is responsible for establishing connections with the requested bandwidths. All transit switches have to be involved in the Bearer Control. The separation of Call and Bearer Control leads to the separation of Call Control Access Function (CCAF) and Bearer Control Access Function (BCAF).

Future multimedia services will require the allocation and on-line modification of special resources such as bridges and converters. Special resources may be both hardware devices and software entities providing functions such as compression, decompression, synchronization, security, multiparty branching and others. To control these specialized resources an additional Resource Control Protocol was proposed in [SIGN] and [COMP].

Quality of Service aims at producing a quantified assessment of user satisfaction. User satisfaction can be quantified according to the network's ability to fulfill service requirements, and the amount and flexibility of operational maintenance offered [E.800]. To maintain the QOS and to provide guaranteed QOS bearer and resource control are involved. For example degrading QOS caused by congestion can trigger rerouting of the call.

An often used example to create multimedia services is to use multi-channel or multi-party calls where different service components are obtained from different service nodes. Different service components can be displayed in different user interface windows which are created by the service application when needed. The question how to control these types of services has been studied in [ISCP] and [SUMM]. In [ISCP] the multimedia services are controlled by a Bridging/Branching node which resembles a present day switch with ISDN User Part. In [SUMM] an intelligent service control point called concept provider service node was introduced to control the multimedia service components.

\section{CALL PROCESSING MODEL}

In the mobile and multimedia services new control functions have been proposed. They are not necessarily user controlled or call related, but they trigger network intelligence depending on their triggering condition. We shall consider here different triggering conditions and the corresponding control functions and how they can be distributed in the network.

In the IN distributed functional plane architecture [Q.1204] the trigger event detection is centralized in the Basic Call State Model in CCF and the corresponding signalling interactions with SCF are managed by SSF. To include other types of triggering conditions and to 
make them communicate with corresponding control functions we propose the following new model.

Let us group trigger types and control function types and let us define signalling message interfaces provided by each control function. From the examples in previous chapters we can introduce the following trigger types:

- Terminal access activated triggering (TAT) - access rights to the network which can be in the terminal or in the network elements.

- QOS activated triggering (QAT) based on monitoring the service or bearer connection quality. Examples of this are

- mobile location updating and handovers,

- multimedia connection rerouting in case of degrading QOS.

- Service component activated triggering (SCAT) based on activating/deactivating or synchronising new service components with needed resource, e.g.

- involvement of decompression resources in case of new service component stream,

- synchronisation of video and audio components.

- Service identifier activated triggering (SIAT) based on the given service identifier (number), such as IN services with given service identifier (freephone with 0800).

- User interaction activated triggering (UIAT) providing control activated by user, such as

- Calling card and voice services,

- Universal Personal Telephony (UPT).

- Service activated triggering (SAT) controlled by the service logic in terminals or network nodes. For example the multimedia service uses another service as its part e.g. to open new window and to display there video segment from some other server.

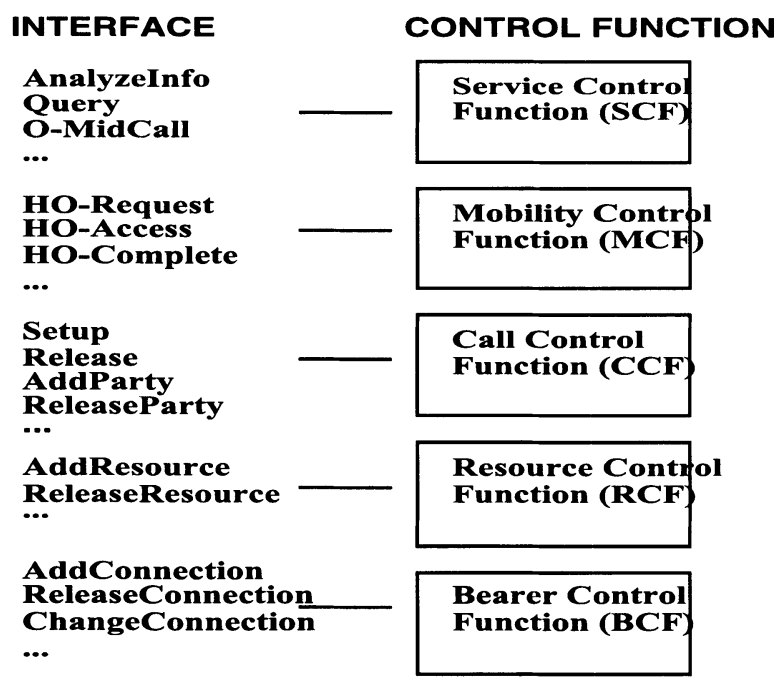

Figure 3 Example of Control Functions and their Interfaces 
Each trigger type may communicate with the corresponding control functions:

- Service Control Function (SCF): SAT, TAT,

- Mobility Control Function (MCF): QAT, SAT, TAT

- Call Control Function (CCF): SIAT, UIAT, SCAT

- Resource Control Function (RCF): SCAT, QAT

- Bearer Control Function (BCF): SCAT, QAT

Here we have separated Mobility Control Function from SCF though in Chapter 3 they were both in SCF (Figure 2). The above control functions provide signalling message interfaces for the triggers (Figure 3). The SCF interface can be based on INAP operations [Q.1218] and the MCF interface can be based on GSM Handover procedures [ETSI].

Triggers may be considered as agents providing access to control functions. Hence we shall call entities providing triggering functions as trigger agents.

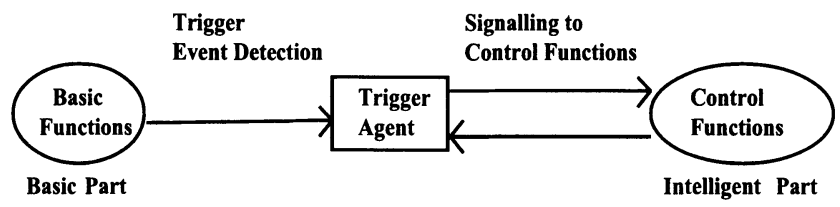

Figure 4 Trigger Agent

The trigger agents monitor the triggering conditions with triggering event detection. When some condition is met, the triggering agent sends signalling messages to the corresponding control functions provided by the network.

Trigger agents are mediators between the basic and additional (intelligent) functions in the network.

Intelligence is specified by giving the available control functions and the triggers which signal to them. The distribution of intelligence is described by placing the trigger and control functions in network entities. Network architecture specification describes how trigger agents and control functions are distributed in network entities.

A service can now be built on top of the basic (generic) control functions by introducing the trigger agents and their interactions with basic and additional control functions available (Figure 5). The trigger event detection can be distributed outside the CCF and the SSF can be considered as the interface specification for CCF based triggers and SCF.

There may be Mediation Points in the network which provide the control function signalling interfaces to Trigger Agents outside the teleoperator management domain and guarantee the separation of operator controlled network from user or service provider controlled network parts [MEDI]. 


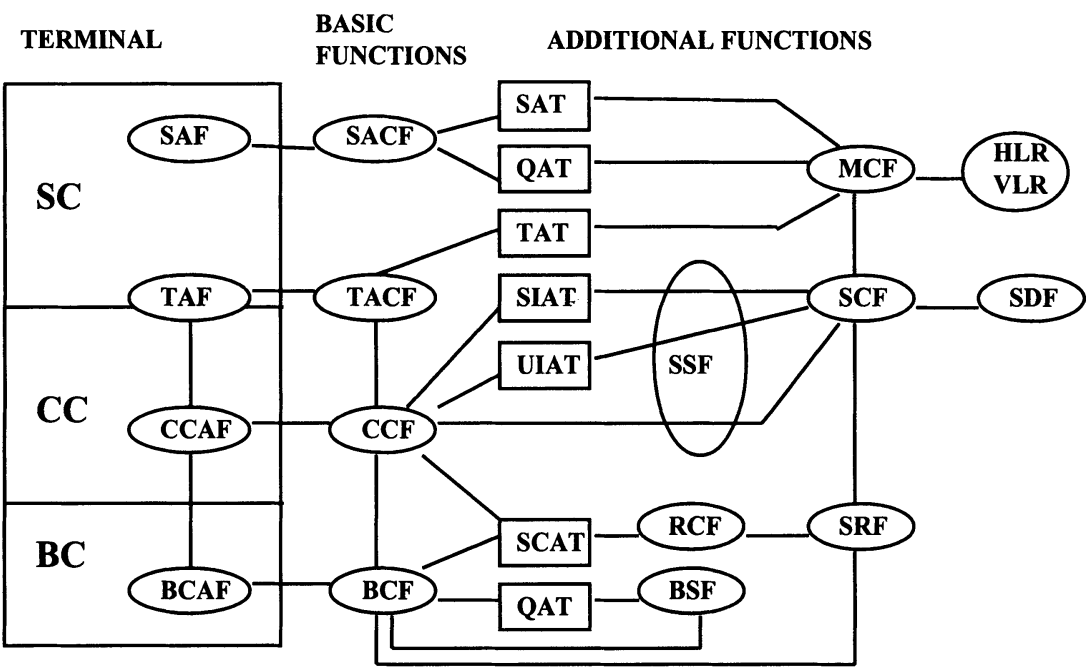

Figure 5 Examples of Control Functions and Triggers attached to them

\section{EXAMPLES}

With multimedia services the user application can have access to different service components with corresponding component SAT agent functions. In the user application the Trigger Agents can be represented by icons and they can be available from service providers (Figure 6).

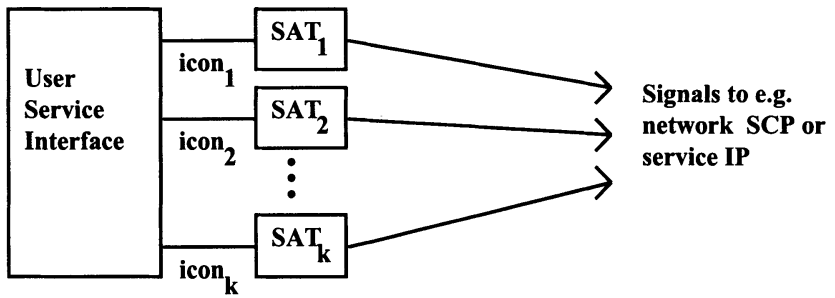

Figure 6 Trigger Agents as icons in user application 
With connection less services the CCF is placed either in the user equipment or in the service nodes. In the next figure we present a connection less mobile service. The terminal in the figure contains some present day base station controller functions.

$\begin{array}{lll}\text { TERMINAL } & \text { ROUTER } & \text { SERVER }\end{array}$

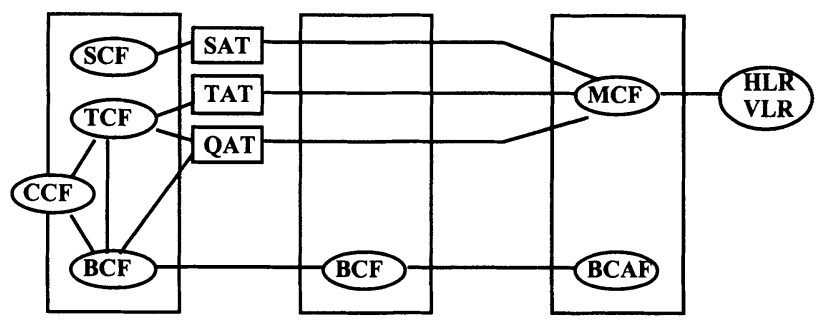

Figure 7 Terminal controlled connection less mobility

Let us finally consider a service provider operating a coordinating Service Control Point (SCP). The SCP is connected to the public network and it controls combinations of other services available in different service providers Service Nodes (SN).
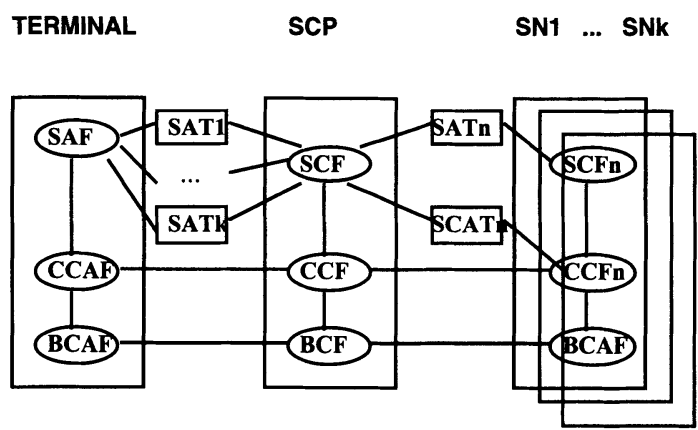

Figure 8 Service combined from several Service Nodes

The access to the separate services $(j=1, . ., k)$ is provided by Trigger Agents SATj, which signal to the coordinating Service Control Function SCF. Each separate service SCFn can in turn invoke other services by Trigger Agent SATn signalling to the coordinating SCF. The coordinating SCP can provide the necessary agents SATj to the users and keep the user database for administration and billing. The use of services is measured by Agents SCATn in each 
Service Node SNn, and the measurement results are signalled to the user database in the coordinating SCP.

\section{REFERENCES}

[AIMN] A. Nakajima, et. al. "Advanced Intelligent Mobile Communications Network", Proc. ISS ‘95, v. 2, April 1995, pp. 320-324.

[AUNI] ATM User-Network Interface Specification, Version 3.0, September 10, 1993.

[COMP] P. Hellemans, G. Reyniers "An Object-Oriented Approach to Controlling Complex Communication Configurations", Proc. ISS ‘95, v. 2, April 1995, pp. 72- 76.

[DATA] L. A. Craven, et. al. "Evolving Subscriber Data Management in Digital Switches to Provide Personal Communications Services", Proc. ISS ‘95, v. 2, April 1995, pp. 31-35.

[E.800] Terms and Definitions Related to the Quality Telecommunications Services, ITU-T Recommendation E.800, 1993.

[ETSI] European digital cellular telecommunications system (Phase 2); Handover Procedures, ETSI GSM 03.09, 1994.

[FUTU] D. Gaiti, G. Pujolle "Which Intelligence in Future Networks", Intelligent Networks, Chapman \& Hall, 1995, pp. 280-290.

[1.374] Framework recommendation on "Network capabilities to support multimedia services", ITU-T Recommendation I.374, 1993.

[ISCP] ISCP Baseline Document, version 3.1, ITU-T Study Group 11 Report R10, June 1993.

[LOCA] H. Hämmäinen, P. Lahtinen "On Location of Service Control", Intelligent Networks, Chapman \& Hall, 1995, pp. 246-253.

[MEDI] W. Heinmiller, et. al. "Solutions for Mediated Access to the Intelligent Network, Proc. ISS ‘95, v. 2, April 1995, pp. 217-221.

[Q.1204] Intelligent Network Distributed Functional Plane Architecture, ITU-T Recommendation Q.1204, 1993.

[Q.1218] Interface Recommendation for Intelligent Networks CS1, ITU-T Recommendation Q.1218, 1993.

[SIGN] S. Tohme', et. al. "Signaling Evolution for B-ISDN", Proc. Telcom 95, SouthAfrica, March 27-29, 1995.

[SUMM] O. Martikainen, et. al. "Comparison of Broadband Intelligent Network Architectures", Intelligent Networks, Chapman \& Hall, 1995, pp. 253-269.

[UMTS] E. Buitenwerf, et. al. "UMTS: Fixed Network Issues and Design Options", IEEE Personal Communications, February 1995. 


\section{BIOGRAPHY}

Konstantin E. Samouylov received the M.Sc. degree in mathematics from the Peoples' Friendship University of Russia (RPFU) in 1978 and the Ph.D. degree in probability and queuing theory from the Moscow State University in 1985. During 1985-1990 he held a position of associate professor at RPFU Faculty of Sciences (Information Technology laboratory). In 1991 he joined RPFU Computer Center where he is presently a vice-director. His current research interests are performance analysis of telecommunication networks, SS\#7 network dimensioning and planning, IN signaling systems portable software development, broadband signaling and multimedia services.

Valeri Naoumov is Computer Center Director Research with People's Friendship University in Moscow, Russia. Areas of Expertise: queuing theory, computational algorithms, traffic models, intelligent network, signalling protocols, telecommunication protocols implementation. He has written many technical articles and two books. V. Naoumov holds Ph.D. in Applied Mathematics from Computer Centre, Russian Academy of Sciences (1979).

Olli Martikainen, Ph.D., from Helsinki University and M.Sc. from Helsinki University of Technology. He has been doing research in several positions in Helsinki University of Technology (1976 - 1982), Oxford University (1980 - 1981) and Technical Research Centre of Finland (1982 - 1985). Later he has been R\&D manager at Nokia Electronics (1985 - 1986), department manager at Nokia Research Centre (1986 - 1988), professor and head of Datacommunications Institute at Lappeenranta University of Technology (1988 - 1989) and professor at Technical Research Centre (1989 - 1991). Since 1991 he has been research director at Telecom Finland. Currently he is vice president, R\&D, at Telecom Finland Ltd., and professor at Helsinki University of Technology. His main areas of interest are telecommunication software methods and tools, network architectures, performance analysis and new industrial and economic structures in telecommunications. 